\title{
A Theoretical Investigation of the Ring Strain Energy, Destabilization Energy, and Heat of Formation of CL-20
}

\author{
John A. Bumpus \\ Department of Chemistry and Biochemistry, University of Northern Iowa, Cedar Falls, IA 50614, USA \\ Correspondence should be addressed to John A. Bumpus, john.bumpus@uni.edu
}

Received 10 April 2012; Accepted 13 August 2012

Academic Editor: Dennis Salahub

Copyright () 2012 John A. Bumpus. This is an open access article distributed under the Creative Commons Attribution License, which permits unrestricted use, distribution, and reproduction in any medium, provided the original work is properly cited.

The cage compound CL-20 (a.k.a., 2,4,6,8,10,12-hexanitro-2,4,6,8,10,12-hexaazaisowurtzitane, HNIW, or 2,4,6,8,10,12-hexanitro2,4,6,8,10,12-hexaazatetracyclo $\left[5 \cdot 5 \cdot 0 \cdot 0^{3,11} \cdot 0^{5,9}\right]$ dodecane) is a well-studied high-energy-density material (HEDM). The high positive gas- $\left(\Delta_{f} H_{g}^{\circ}\right)$ and solid- $\left(\Delta_{f} H_{s}^{\circ}\right)$ phase heat of formation values for CL-20 conformers have often been attributed to the strain energy of this cage compound and, by implication, to the conventional ring strain energy (CRSE) inherent in isowurtzitane which may be viewed as a "parent compound" (although not the synthetic precursor) of CL-20. $\Delta_{f} H_{g}^{\circ}$ values and destabilization energies (DSEs), which include the contribution from CRSE, were determined by computation using a relatively new multilevel $a b$ intio model chemistry. Compared to cubane, isowurtzitane does not have an exceptionally high CRSE. It is about the same as that of cyclopropane and cyclobutane. These investigations demonstrate that instead of the CRSE inherent in the isowurtzitane parent compound, the relatively high $\Delta_{f} H_{g}^{\circ}$ and DSE values of CL-20 conformers must be due, primarily, to torsional strain (Pitzer strain), transannular strain (Prelog strain), and van der Waals interactions that occur due to the presence of the six $>\mathrm{N}-\mathrm{NO}_{2}$ substituents that replace the six methylene $\left(-\mathrm{CH}_{2}-\right)$ groups in the isowurtzitane parent compound. These conclusions are even more pronounced when 2,4,6,8,10,12-hexaazaisowurtzitane is viewed as the "parent compound."

\section{Introduction}

The cage compound CL-20 (a.k.a., 2,4,6,8,10,12-hexanitro-2,4,6,8,10,12-hexaazaisowurtzitane, HNIW, or 2,4, 6,8,10,12-hexanitro-2,4,6,8,10,12-hexaazatetracyclo $\quad[5.5 .0$. $0^{3,11} \cdot 0^{5,9}$ d dodecane) is a high-energy-density material (HEDM) that has been developed and studied during the past several years (see Scheme 1).

This compound was first synthesized in 1987 by scientists at the China Lake Naval Weapons Center [1,2]. There is substantial interest in the use of CL-20 as a high explosive and many of its properties (density $=\sim 2 \mathrm{~g} \mathrm{~cm}^{-3}$, detonation velocity $=9.4 \mathrm{~mm} \mathrm{~ms}^{-1}$, oxygen balance $=-11$, detonation pressure $=420 \mathrm{kbar}$, etc.) suggest that it is superior to $\mathrm{RDX}$ (1,3,5-trinitroperhydro-1,3,5-triazine) and HMX (octahydro-1,3,5,7-tetranitro-1,3,5,7-tetrazocine), two high explosives that are widely used at the present time [3-6] (see Scheme 2).
In addition to its use as a high explosive compound, [3] CL-20 has potential use as a propellant [6].

CL-20 is often compared to octanitrocubane (ONC) which, like CL-20, is a polynitro cage compound. Although both have many properties that recommend them for use as high explosives [7-12], economic considerations appear to limit the large scale production and usefulness of ONC $[11,12]$. On the other hand such problems for largescale production of CL-20 appear to have been successfully addressed, although cost reduction is still an issue. CL20 is produced commercially by ATK-Thiokol Propulsion (Brigham City, UT, USA) [13].

The relatively high heat of combustion and heat of detonation values of many HEDMs are due, in part, to their high positive heat of formation values. Octanitrocubane $(\mathrm{ONC})$ is a case in point (see Scheme 3 ).

Estimates of gas-phase $\left(\Delta_{f} H_{g}^{\circ}\right)$ and solid-phase $\left(\Delta_{f} H_{s}^{\circ}\right)$ heat of formation values of this compound have been 


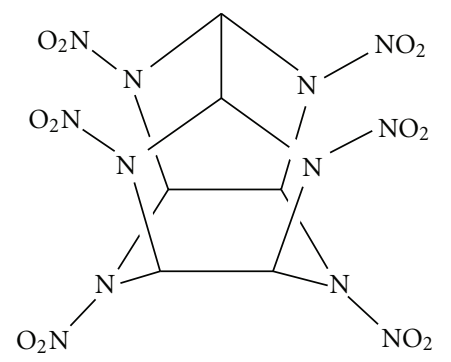

CL-20

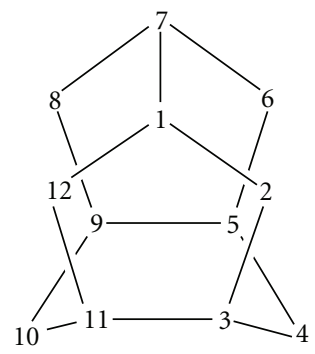

Numbering system for CL-20 and other substituted isowurtzitanes

SCHEMe 1<smiles>O=[N+]([O-])N1CN([N+](=O)[O-])CN([N+](=O)[O-])C1</smiles>

RDX

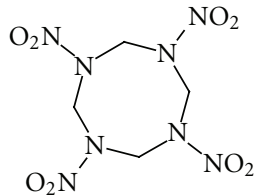

HMX
Scheme 2: Structures of RDX and HMX.

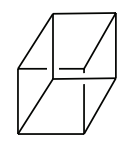

Cubane

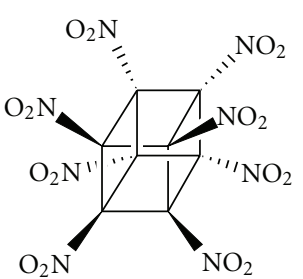

Octanitrocubane

(ONC)
Scheme 3: Structures of cubane and octanitrocubane.

calculated to be $\sim 726$ and $\sim 594 \mathrm{~kJ} / \mathrm{mol}$, respectively $[8,9]$, and it is reasonable to conclude that such high heat of formation values are due, in large measure, to the considerable conventional ring strain energy (CRSE) inherent in the parent compound, cubane.

At first glance, it may appear that, like cubane, isowurtzitane and/or 2,4,6,8,10,12-hexaazaisowurtzitane may also

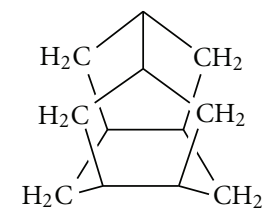

Isowurtzitane

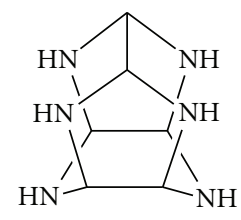

$2,4,6,8,10,12$ Hexaazaisowurtzitane
Scheme 4: Structures of isowurtzitane and 2,4,6,8,10,12-hexaazaisowurtzitane. have very high CRSEs that contribute to the high heat of formation of CL-20 (see Scheme 4).

In this study it is shown that this is not the case. Using gas-phase calculations, it was demonstrated that the CRSE inherent in isowurtzitane is relatively minor (compared to cubane, e.g., which is clearly highly strained) and the CRSEs of 2,4,6,8,10,12-hexaazaisowurtzitane conformers are exceptionally small. Thus the modestly high (compared to ONC) destabilization energy (DSE) and heat of formation values of CL-20 must be due to factors other than the CRSE inherent in the parent compound(s).

\section{Computational Approach}

2.1. Heat of Formation. Gas-phase heat of formation values were calculated using a recently described [15] multilevel ab initio model chemistry (known as T1) as implemented in the Spartan 10 (Wavefunction, Inc., Irvine, CA, USA) suite of programs. The T1 program has been described in substantial detail by Ohlinger et al. [15]. Briefly this program is based on the G3MP2 model chemistry. The major differences between T1 and G3MP2 model chemistries are those in the T1 program; the MP2/6-31G(d) equilibrium geometry is substituted with an $\mathrm{HF} / 6-31 \mathrm{G}(\mathrm{d})$ equilibrium geometry. The HF/6-31G(d) frequency and $\operatorname{QCISD}(\mathrm{T}) / 6$ $31 \mathrm{G}(\mathrm{d})$ energy steps are removed and the MP2/G3MP2 large energy step is approximated using a dual basis set RI-MP2 technique [15].

Comparing computed results with experimental values from 1805 structurally diverse compounds from the NIST database, Ohlinger et al. [15] reported this procedure to have a mean absolute error of $8.5 \mathrm{~kJ} / \mathrm{mol}$ and a root mean square error of $11.5 \mathrm{~kJ} / \mathrm{mol}$. Thus it is reasonable to suggest that the values computed in this investigation have similar accuracy.

2.2. Conventional Ring Strain Energy (CRSE) and Destabilization Energy (DSE). Conventional ring strain energies (CRSE) as described by Benson and associates $[16,17]$ were calculated by difference using gas-phase heat of formation $\left(\Delta_{f} H^{\circ}\right.$ (grp. add.) $)$ values determined by group additivity theory (without consideration of ring strain energy, etc.) and the gas-phase heat of formation $\left(\Delta_{f} H_{g}^{\circ}\right)$ of the molecule determined by experiment or by computation, using theory 
(such as T1 or G3MP2) that is sufficiently robust to account for and include ring strain and other contributing factors. Thus, for gas-phase reactions, CRSE is calculated as follows:

$$
\text { CRSE }=\Delta_{f} H_{g}^{\circ}-\Delta_{f} H^{\circ} \text { (grp. add.)g. }
$$

Leroy [18] makes the point that for unsubstituted cyclic compounds, destabilization energy (Leroy uses the term negative stabilization energy) is equivalent to CRSE. Thus in this study the term CRSE is used to describe strain inherent in the unsubstituted cyclic and cage compounds under investigation. In contrast to CRSE, the term destabilization energy (DSE) is used here as a more inclusive term and is reserved for characterization of substituted cyclic and cage compounds although it is calculated in precisely the same way as CRSE:

$$
\text { DSE }=\Delta_{f} H_{g}^{\circ}-\Delta_{f} H^{\circ} \text { (grp. add.) } g .
$$

Although the distinction between CRSE and DSE is somewhat arbitrary, it is warranted as it should be appreciated that for substituted cyclic and cage compounds DSE includes not only the energy contribution from ring strain but also the effects that substituents impose on the structure. For example, substituents, such as nitro groups, may increase or decrease bond angles and bond lengths (depending on placement). Furthermore van der Waals interactions between adjacent or nearby substituents will affect DSE. For nitro groups, close interaction leads to an increase in DSE. Other factors such as torsional strain (Pitzer strain) and transannular strain (Prelog strain) also contribute to DSE.

It should also be appreciated that DSE may be calculated for acyclic compounds in which CRSE, by definition, is not a factor.

The ASTM CHETAH 8.0 software program (CHETAH (The Computer Program for Chemical Thermodynamics and Energy Release Evaluation) University of South Alabama, Mobile Alabama 36688-0002 (http://www.southalabama.edu/engineering/chemical/ chetah/index.html) and ASTM International (http://www. astm.org/)) was used to calculate strainless gas-phase heat of formation values $\left(\Delta_{f} H^{\circ}\right.$ (grp. add.) $\left.g\right)$ that, in turn, were used to calculate CRSE and DSE, as described above. This software uses the Benson group additivity approach. Thus the Benson group values as well as other group values provided by the software manufacturer were used in these calculations $[16,17,19]$.

2.3. Contribution of Ring Nitrogen and Nitro Group Substituents to the DSE, $\left(\Delta_{f} H^{\circ}{ }_{(g r p . ~ a d d .) g}\right)$, and $\Delta_{f} H_{g}^{\circ}$ Values of $C L-20$. The contributions of ring nitrogen and nitro group substituents to the DSE and $\Delta_{f} H_{g}^{\circ}$ values of CL-20 were determined using the T1 multilevel ab initio model chemistry [15]. To do this, the $\Delta_{f} H_{g}^{\circ}$ value for CL-20 conformer HNIW-III (one of four known conformers of CL-20 See [14]) was calculated first. The $\Delta_{f} H_{g}^{\circ}$ value for $2,4,6,8,10$ pentanitro-2,4,6,8,10,12-hexaazaisowurtzitane was then calculated following computational removal of one of the nitro groups from the equilibrium conformation of HNIW-III.
$\Delta_{f} H_{g}^{\circ}$ values for the remaining nitrohexaazaisowurtzitanes were calculated in a similar manner. The same approach was used to assess the effect of ring nitrogen substitution on $\Delta_{f} H_{g}^{\circ}$. Nitro groups or ring nitrogens were computationally removed in the following order $\mathrm{N}-12, \mathrm{~N}-10, \mathrm{~N}-8, \mathrm{~N}-6, \mathrm{~N}-4$, and $\mathrm{N}-2 . \Delta_{f} H^{\circ}$ (grp. add.)g values were also calculated using the group additivity approach described above and DSE values were calculated using (2). All $\Delta_{f} H_{g}^{\circ}, \Delta_{f} H^{\circ}$ (grp. add.) g, and DSE values refer to such values at $298.15 \mathrm{~K}$.

For comparison, the effect of nitro group substituents on $\Delta_{f} H_{g}^{\circ}$ and DSE values for nitrocubane and polynitrocubanes was determined in a similar manner. In these calculations, the $\Delta_{f} H_{g}^{\circ}$ and DSE values for octanitrocubane were calculated first. The $\Delta_{f} H_{g}^{\circ}$ and DSE values for heptanitrocubane were then calculated following computational removal of one of the nitro groups from the equilibrium conformation of octanitrocubane. $\Delta_{f} H_{g}^{\circ}$ and DSE values for the remaining nitrocubanes were calculated in a similar manner. In addition to octanitrocubane and heptanitrocubane, $\Delta_{f} H_{g}^{\circ}$ and DSE values were calculated for 1,2,3,4,5,6-hexanitrocubane, 1,2,3,4,5-pentanitrocubane, 1,2,3,8-tetranitrocubane, 1,2,3trinitrocubane, 1,2-dinitrocubane, and nitrocubane.

\section{Results and Discussion}

3.1. Conventional Ring Strain Energy (CRSE) and Heat of Formation. Computed values for gas-phase heat of formation $\left(\Delta_{f} H_{g}^{\circ}\right)$, strainless heat of formation $\left(\Delta_{f} H^{\circ}\right.$ (grp. add.)g $)$, and destabilization energy (DSE) for four CL-20 conformations as well as several other compounds directly relevant to this investigation are presented in Table 1.

There are several polymorphs (conformers) possible for CL-20 [11, 14, 20-26]. To date five polymorphs have been isolated experimentally and four have been studied computationally. Zhou et al. [14] used density functional theory at the B3LYP/6-31G(d,p) level to characterize four CL-20 conformations. In Zhou et al.'s [14] terminology, conformations HNIW-I, HNIW-II, HNIW-III, and HNIWIV represent local minima on the potential energy surface. Zhou et al.'s [14] terminology is used here (Figure 1).

The computed gas-phase heat of formation values of the four CL-20 conformations (HNIW-I, HNIW-II, HNIWIII, and HNIW-IV) range between 548.0 and $571.6 \mathrm{~kJ} / \mathrm{mol}$. Subtracting the heat of sublimation value $(168.7 \mathrm{~kJ} / \mathrm{mol})$ for CL-20 calculated by Zeman and Krupka [27] yields solid phase heat of formation values for these conformations ranging from 379.3 to $402.9 \mathrm{~kJ} / \mathrm{mol}$. These values are clearly consistent with the experimentally determined solid phase heat of formation values $(377.4 \pm 13$ and $431.0 \pm 13 \mathrm{~kJ} / \mathrm{mol})$ reported for two of the crystalline polymorphs [5].

Isowurtzitane and 2,4,6,8,10,12-hexaazaisowurtzitane may be thought of as the parent compounds (although not the synthetic precursors) of CL-20. Similarly cubane may be thought of as the parent compound for ONC. Thus it was informative to compute the $\Delta_{f} H_{g}^{\circ}$ values, $\Delta_{f} H^{\circ}$ (grp. add.) $g$ values, and CRSE values for these compounds to determine and compare how the cage structures of the parent 
TABLE 1: Comparison of the strainless gas-phase heat of formation, destabilization energy, and gas-phase heat of formation of CL-20, ONC, and other relevant compounds of interest.

\begin{tabular}{|c|c|c|c|c|}
\hline Compound & $\begin{array}{l}\text { Strainless heat of formation } \\
\qquad \Delta_{f} H_{\text {(grp. add.)g }}^{\circ}(\mathrm{kJ} / \mathrm{mol})\end{array}$ & $\begin{array}{l}\text { Heat of formation } \\
\Delta_{f} H_{g}^{\circ}(\mathrm{kJ} / \mathrm{mol})\end{array}$ & $\begin{array}{l}\text { Destabilization energy } \\
\text { or CRSE }(\mathrm{kJ} / \mathrm{mol})\end{array}$ & $\begin{array}{l}\text { Destabilization energy per } \mathrm{C}+\mathrm{N} \\
\text { present in the ring or the cage } \\
(\mathrm{kJ} / \mathrm{mol})\end{array}$ \\
\hline \multirow{3}{*}{ Cyclopropane } & \multirow{3}{*}{-61.9} & 55.8 & 117.7 & 39.2 \\
\hline & & $(39.3)$ & (101.2) & $(33.7)$ \\
\hline & & $(53.3)$ & $(115.2)$ & $(38.4)$ \\
\hline \multirow{2}{*}{ Cyclobutane } & \multirow{2}{*}{-82.5} & 29.7 & 112.2 & 28.1 \\
\hline & & $(27.7)$ & $(110.2)$ & $(27.6)$ \\
\hline HAIW I & 325.3 & 306.6 & -18.7 & -1.6 \\
\hline HAIW II & 325.3 & 301.7 & -23.6 & -2.0 \\
\hline HAIW III & 325.3 & 323.1 & -2.2 & -0.2 \\
\hline HAIW IV & 325.3 & 290.5 & -34.8 & -2.9 \\
\hline Isowurtzitane & -171.5 & -63.5 & 108.0 & 9.0 \\
\hline CL-20 (HNIW I) & 240.0 & 558.8 & 318.8 & 26.6 \\
\hline CL-20 (HNIW II) & 240.0 & 554.4 & 314.4 & 26.2 \\
\hline CL-20 (HNIW III) & 240.0 & 571.6 & 331.6 & 27.6 \\
\hline CL-20 (HNIW IV) & 240.0 & 548.0 & 308.0 & 25.7 \\
\hline \multirow{2}{*}{ Cubane } & \multirow{2}{*}{-63.6} & 622.2 & 685.8 & 85.7 \\
\hline & & $(622.2)$ & 685.8 & 85.7 \\
\hline \multirow{2}{*}{ Octanitrocubane } & \multirow{2}{*}{-405.0} & 687.5 & $1,092.5$ & 136.6 \\
\hline & & $(726)$ & $(1,131)$ & $(141)$ \\
\hline
\end{tabular}

The values in parentheses for octanitrocubane are the heat of formation value calculated by Zhang and Xiao [8] and values calculated using this heat of formation.

Other values in parentheses are those reported in, or calculated using, those referenced in the NIST Chemistry WebBook (http://webbook.nist.gov/chemistry/) or in the CRC's Standard Thermodynamic Properties of Chemical Substances.

HAIW refers to 2,4,6,8,10,12-hexaazaisowurtzitane.

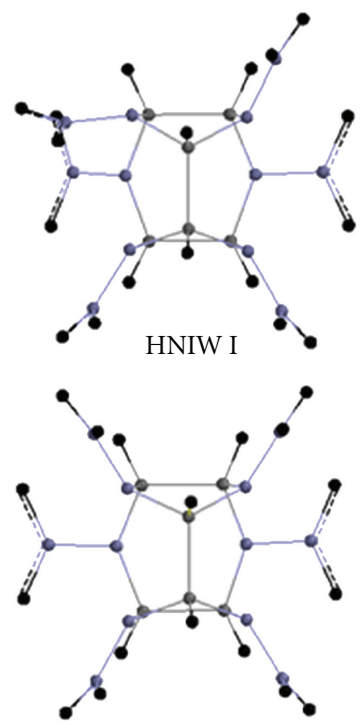

HNIW III

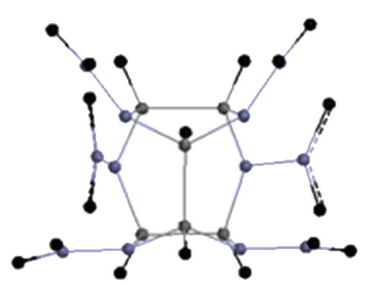

HNIW II

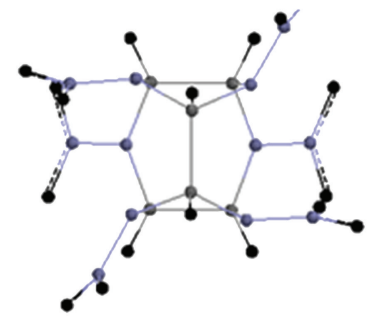

HNIW IV
Figure 1: Conformations of CL-20 described by Zhou et al. [14].

compounds may contribute to the $\Delta_{f} H_{g}^{\circ}$ values of CL-20 and ONC. These values are also presented in Table 1.
One characteristic of ONC that makes it a high-energy compound is the fact that the cubane cage imposes tremendous ring strain on the compound and it is the ring strain that is inherent in the cubane cage that imparts a very substantial contribution to the $\Delta_{f} H_{g}^{\circ}$ and DSE of ONC. Several experimental and computed values for the CRSE (destabilization energy) of cubane have been reported (see [28] and references therein). These values range between 656.9 and $707.6 \mathrm{~kJ} / \mathrm{mol}$ and are consistent with the value $(685.8 \mathrm{~kJ} / \mathrm{mol})$ calculated in this investigation (Table 1$)$. It should also be appreciated that substitution of the eight hydrogens in cubane by eight nitro groups to form ONC leads to a more negative $\Delta_{f} H^{\circ}$ (grp. add.)g, and this contributes substantially to the exceptionally high DSE of ONC.

Because ONC and CL-20 are both polynitro cage compounds, it is tempting to assume that the high heat of formation values characteristic of both compounds are due, in large measure, to ring strain inherent in the parent cage compounds (i.e., their CRSE). This is clearly true for ONC. However, this is not the case for CL-20. Data presented in Table 1 shows that the calculated CRSE (destabilization energy) of isowurtzitane is only $108.0 \mathrm{~kJ} / \mathrm{mol}$. Although this value is not inconsequential (it is about the same as that observed for cyclopropane and cyclobutane), it is much less than the CRSE of cubane $(\mathrm{CRSE}=685.8 \mathrm{~kJ} / \mathrm{mol})$, a compound that clearly deserves to be called "highly strained." 


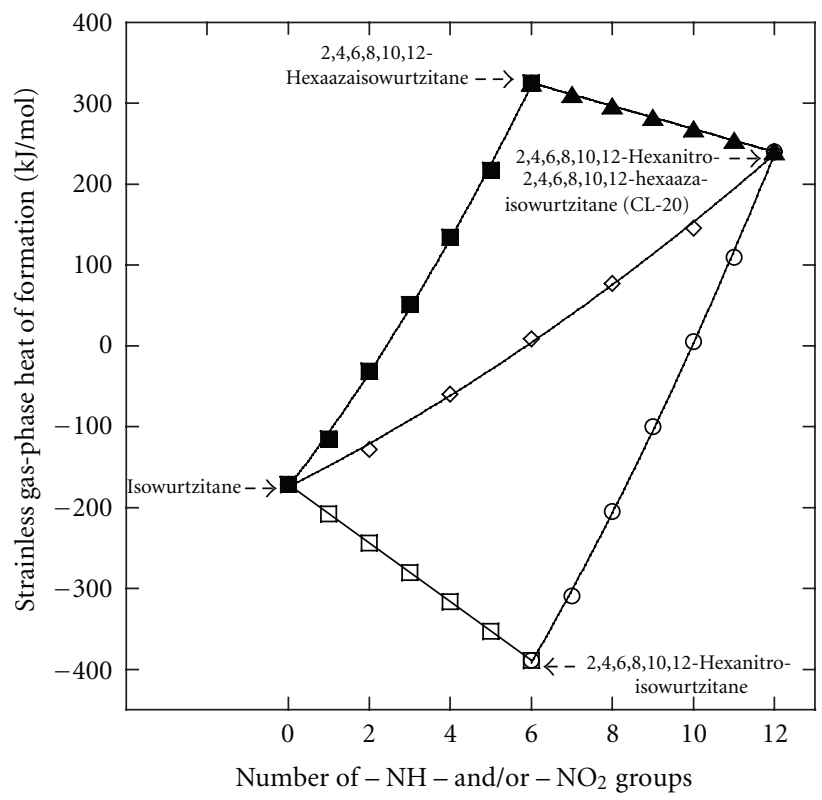

(a)

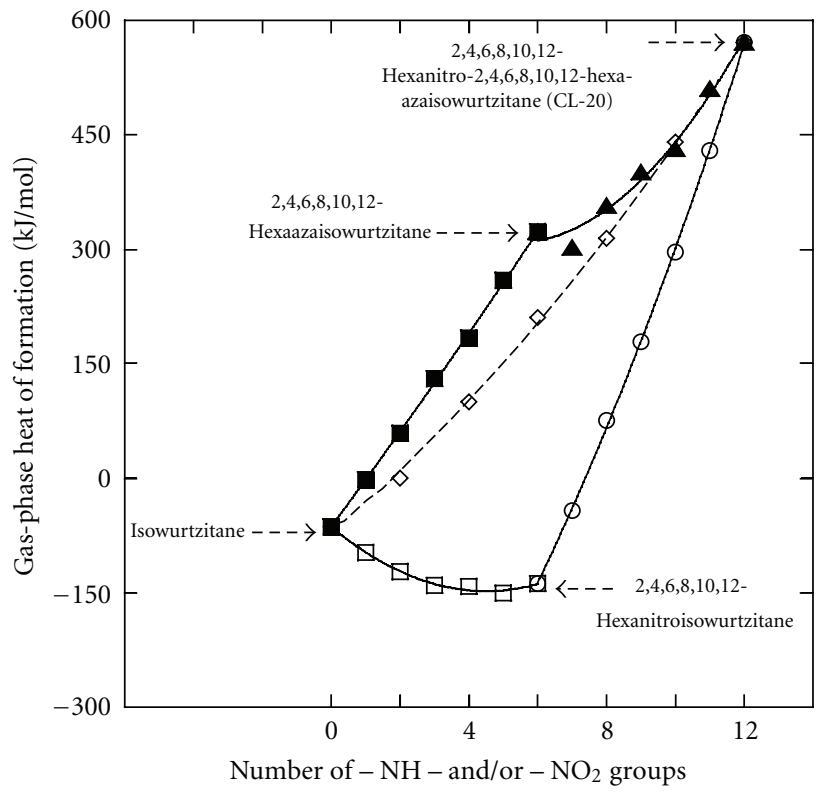

(b)

Figure 2: (a) Effect of $-\mathrm{NH}-$ and $-\mathrm{NO}_{2}$ substitution on the strainless gas-phase heat of formation of isowurtzitane. Closed squares: effect on the strainless heat of formation of adding - NH- groups to isowurtzitane in the 2, 4, 6, 8, 10 and 12 positions, open squares: effect on the strainless heat of formation of adding $-\mathrm{NO}_{2}$ groups to isowurtzitane in the 2, 4, 6, 8, 10 and 12 positions, open circles: effect on the strainless heat of formation of adding-NH- groups to 2,4,6,8,10,12-hexanitroisowurtzitane in the 2, 4, 6, 8, 10 and 12 positions, closed triangles: Effect on the strainless heat of formation of adding $-\mathrm{NO}_{2}$ groups to 2,4,6,8,10,12-hexaazaisowurtzitane in the 2, 4, 6, 8, 10 and 12 positions, tilted open squares: effect on the strainless heat of formation of adding $>\mathrm{N}-\mathrm{NO}_{2}$ groups to isowurtzitane in the $2,4,6,8,10$ and 12 positions. (b) Effect of $-\mathrm{NH}-$ and $-\mathrm{NO}_{2}$ substitution on the gas phase heat of formation of isowurtzitane calculated using the T1 multilevel $a b$ initio model chemistry approach described by Ohlinger et al. [15]. Closed squares: effect on the heat of formation of adding-NH-groups to isowurtzitane in the 2, 4, 6, 8, 10 and 12 positions, Open squares: Effect on the heat of formation of adding $-\mathrm{NO}_{2}$ groups to isowurtzitane in the 2, 4, 6, 8, 10 and 12 positions, Open circles: Effect on the heat of formation of adding -NH- groups to 2,4,6,8,10,12-hexanitroisowurtzitane in the 2, 4, 6, 8, 10 and 12 positions, Closed triangles: effect on the heat of formation of adding $-\mathrm{NO}_{2}$ groups to 2,4,6,8,10,12-hexaazaisowurtzitane in the 2, 4, 6, 8, 10 and 12 positions, tilted open squares: effect on the heat of formation of adding $>\mathrm{N}-\mathrm{NO}_{2}$ groups to isowurtzitane in the $2,4,6,8,10$ and 12 positions.

This comparison is even more striking when calculated CRSEs are expressed on a per carbon basis. When analyzed in this manner, the CRSE for isowurtzitane per carbon is even less than that observed for cyclopropane and cyclobutane and almost ten times less than that observed for cubane (Table 1).

An even more appropriate comparison involves 2,4,6,8,10,12-hexaazaisowurtzitane. When CRSEs (destabilization energies) for four conformers of 2,4,6,8,10,12hexaazaisowurtzitane (derived from HNIW-I, HNIW-II, HNIW-III, and HNIW-IV) were calculated, values were found to range from -34.8 to $-2.2 \mathrm{~kJ} / \mathrm{mol}$. Negative CRSE or DSE values are more properly known as stabilization energies (SEs) and are clearly not responsible for the high $\Delta_{f} H_{g}^{\circ}$ and DSE of the CL-20 conformers.

3.2. Effect of $-\mathrm{NH}-$ and $-\mathrm{NO}_{2}$ Substitutents on CRSE, DSE and Heat of Formation. Figures 2(a) and 2(b) present the relative effects of successive $-\mathrm{NH}-,-\mathrm{NO}_{2}$ and $>\mathrm{N}-\mathrm{NO}_{2}$ group substitutions on the heat of formation of isowurtzitane.

In Figure 2(a), only the strainless group contribution to gas phase heat of formation $\left(\Delta_{f} H^{\circ}\right.$ (grp. add.)g $)$ values is considered. Substitution of hydrogens in isowurtzitane by $-\mathrm{NO}_{2}$ groups (open squares) actually contributes to a decrease in $\Delta_{f} H^{\circ}$ (grp. add.)g values. Conversely, substitution of nitrogen for carbon atoms in isowurtzitane (closed squares) results in an increase of $\Delta_{f} H^{\circ}$ (grp. add.)g values. A similar analysis can also be accomplished by substituting methylene $\left(-\mathrm{CH}_{2}-\right)$ groups with $>\mathrm{N}-\mathrm{NO}_{2}$ groups. This, of course, is the equivalent of simultaneously substituting a carbon and two hydrogens with a nitrogen and an $-\mathrm{NO}_{2}$ group, respectively. Figure 2(a) demonstrates that substitution of methylene $\left(-\mathrm{CH}_{2}-\right)$ groups with $>\mathrm{N}-\mathrm{NO}_{2}$ groups results in an increase in strainless $\Delta_{f} H^{\circ}$ (grp. add.)g values (tilted open square).

In Figure 2(b) a similar analysis is presented using $\Delta_{f} H_{g}^{\circ}$ values computed using the $\mathrm{T} 1 a b$ initio procedure.

Results (Figure 2(b)) demonstrate that substitution of hydrogens in isowurtzitane by $-\mathrm{NO}_{2}$ decreases $\Delta_{f} H_{g}^{\circ}$ values (open squares) while nitrogen substitution increases $\Delta_{f} H_{g}^{\circ}$ values (closed squares). When the hydrogens bonded to nitrogens in 2,4,6,8,10,12-hexaazaisowurtzitane are substituted by $-\mathrm{NO}_{2}$ groups, the $\Delta_{f} H_{g}^{\circ}$ value of the first compound in this series is decreased. However, subsequent substitutions of hydrogen by $-\mathrm{NO}_{2}$ result in increased $\Delta_{f} H_{g}^{\circ}$ values 


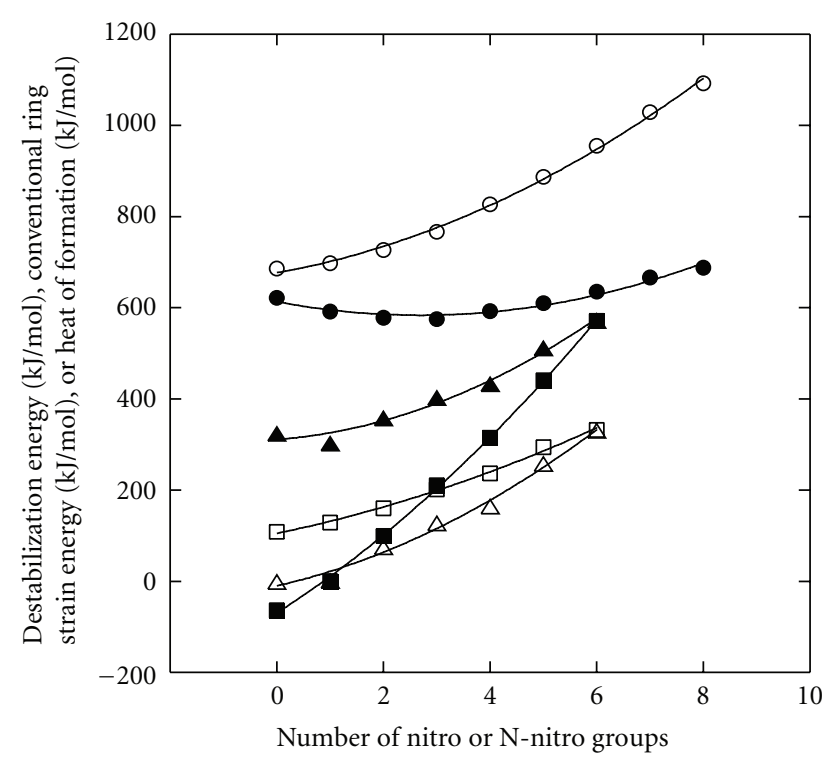

FIgURE 3: The effect on DSE and $\Delta_{f} H_{g}^{\circ}$ of substituting $-\mathrm{NO}_{2}$ groups for hydrogens in $>\mathrm{C}-\mathrm{H}$ groups in cubane compared with (1) the effect of substituting $-\mathrm{NO}_{2}$ groups for hydrogens in $>\mathrm{N}-\mathrm{H}$ groups in 2,4,6,8,10,12-hexaazaisowurtizane and (2) the effect of substituting $-\mathrm{CH}_{2}$-groups in isowurtzitane with $>\mathrm{N}-\mathrm{NO}_{2}$ groups. Note: for compounds having no $-\mathrm{NO}_{2}$ substituents, CRSE = DSE. Open circles: CRSE and DSE values for cubane and nitrocubanes, open triangles: CRSE and DSE values for 2,4,6,8,10,12-hexaazaisowurtzitane and $-\mathrm{NO}_{2}$ substituted 2,4,6,8,10,12-hexaazaisowurtzitanes, open squares: CRSE and DSE values for isowurtzitane and $>\mathrm{N}-\mathrm{NO}_{2}$ substituted isowurtzitane (i.e., nitroazaisowurtzitanes). Closed circles: $\Delta_{f} H_{g}^{\circ}$ of cubane and nitrocubanes, closed triangles: $\Delta_{f} H_{g}^{\circ}$ of 2,4,6,8,10,12-hexaazaisowurtzitane and $-\mathrm{NO}_{2}$-substituted 2,4 , 6,8,10,12-hexaazaisowurtzitanes, closed squares: $\Delta_{f} H_{g}^{\circ}$ of isowurtzitane and $>\mathrm{N}-\mathrm{NO}_{2}$-substituted isowurtzitanes (i.e., nitroazaisowurtzitanes).

(closed triangles). When substitution of methylene groups by $>\mathrm{N}-\mathrm{NO}_{2}$ groups is considered, results demonstrate that $\Delta_{f} H_{g}^{\circ}$ values increase as a result of each substitution (tilted open squares).

The major point of comparison between Figures 2(a) and 2(b) involves the effect of substituting $-\mathrm{NO}_{2}$ groups for hydrogens on nitrogens in 2,4,6,8,10,12hexaazaisowurtzitane. In Figure 2(a), such substitutions result in a decrease in $\Delta_{f} H^{\circ}$ (grp. add.)g (closed triangles), whereas in Figure 2(b) this substitution results, initially, in a decrease of $\Delta_{f} H_{g}^{\circ}$ (closed triangles), but as more $-\mathrm{NO}_{2}$ groups are added, a substantial increase is observed due to a combination of torsional strain (Pitzer strain), transannular strain (Prelog strain), and van der Waals interactions between the six $>\mathrm{N}-\mathrm{NO}_{2}$ substituents.

It should be pointed out that such strain and interactions result because, in contrast to noncage or acyclic compound, the parent cage compounds presents a rigid scaffold that substantially limits bond rotation to relieve or minimize strain, and van der Waals interactions.
Finally, the effect on DSE and $\Delta_{f} H_{g}^{\circ}$ of substituting $-\mathrm{NO}_{2}$ groups for hydrogens in $>\mathrm{C}-\mathrm{H}$ groups in cubane is compared with the effect of substituting $-\mathrm{NO}_{2}$ groups for hydrogens in $>\mathrm{N}-\mathrm{H}$ groups in 2,4,6,8,10,12-hexaazaisowurtizane (Figure 3).

Also presented and compared (Figure 3 ) is the effect on DSE and $\Delta_{f} H_{g}^{\circ}$ caused by substituting $-\mathrm{CH}_{2}$-groups in isowurtzitane with $>\mathrm{N}-\mathrm{NO}_{2}$ groups. In all cases, these substitutions increase the DSE relative to the parent compounds and the preceding compound in these substitution series. Figure 3 also nicely demonstrates that, relative to cubane, the CRSE inherent in isowurtzitane and 2,4,6,8,10,12hexaazaisowurtzitane have a comparatively minor effect on the DSE (and therefore on the $\Delta_{f} H_{g}^{\circ}$ ) of CL-20.

\section{Conclusions}

It is known that the high $\Delta_{f} H_{g}^{\circ}$ and DSE values characteristic of octanitrocubane are due, in large measure, to the high $\Delta_{f} H_{g}^{\circ}$ and CRSE values inherent in and characteristic of cubane, its highly strained parent compound.

The situation for CL-20, however, is different. Isowurtzitane and 2,4,6,8,10,12-hexaazaisowurtzitane can be viewed as parent compounds (but not synthetic precursors) of CL20 , but neither of these compounds have exceptionally high CRSE values. This is due to the fact that the bond angles in these compounds are not as highly strained as the $90^{\circ}$ CCC bond angles in cubane and ONC.

Thus the high $\Delta_{f} H_{g}^{\circ}$ and modestly high DSE values characteristic of CL-20 conformers are not due to high CRSE values of the parent compound(s). Instead, the high $\Delta_{f} H_{g}^{\circ}$ and modestly high DSE values of CL-20 conformers must be due, in large measure, to torsional strain (Pitzer strain), transannular strain (Prelog strain), and van der Waals interactions that occur due to the presence of the six $>\mathrm{N}-\mathrm{NO}_{2}$ substituents that are present in this HEDM. Such strain and interactions result or are enhanced because the parent cage compounds presents a rigid scaffold that substantially limits bond rotation that would otherwise relieve or minimize strain and van der Waals interactions. It should be appreciated that the group contribution energies characteristic of the $>\mathrm{N}-\mathrm{NO}_{2}$ groups provide the greatest contribution to the $\Delta_{f} H_{g}^{\circ}$ and DSE of CL-20.

\section{Acknowledgment}

The author wishes to thank the Environmental Sciences Program at the University of Northern Iowa for its continuing support.

\section{References}

[1] A. T. Nielsen, "Caged polynitramine compound," Chemical Abstracts, vol. 128, article 36971t, 1998, U. S. Department of Navy, U. S. Patent Office Application Case No. 70631, June 1987, U. S. Patent Application No. 253, 106, September 1988, U. S. Patent No. 5, 693, 794 (CI 540-554, C07D259/00, December 1997. 
[2] A. T. Nielsen, A. P. Chafin, S. L. Christian et al., "Synthesis of polyazapolycyclic caged polynitramines," Tetrahedron, vol. 54, no. 39, pp. 11793-11812, 1998.

[3] M. Geetha, U. R. Nair, D. B. Sarwade, G. M. Gore, S. N. Asthana, and H. Singh, "Studies on CL-20: the most powerful high energy material," Journal of Thermal Analysis and Calorimetry, vol. 73, no. 3, pp. 913-922, 2003.

[4] V. D. Ghule, P. M. Jadhav, R. S. Patil, S. Radhakrishnan, and T. Soman, "Quantum-chemical studies on hexaazaisowurtzitanes," Journal of Physical Chemistry A, vol. 114, no. 1, pp. 498-503, 2010.

[5] R. L. Simpson, P. A. Urtiew, D. L. Ornellas, G. L. Moody, K. J. Scribner, and D. M. Hoffman, "CL-20 performance exceeds that of HMX and its sensitivity is moderate," Propellants, Explosives, Pyrotechnics, vol. 22, no. 5, pp. 249-255, 1997.

[6] D. Mueller, "New gun propellant with CL-20," Propellants, Explosives, Pyrotechnics, vol. 24, no. 3, pp. 176-181, 1999.

[7] M.-X. Zhang, P. E. Eaton, and R. Gilardi, "Hepta- and octanitrocubanes," Angewandte Chemie International Edition, vol. 39, pp. 401-404, 2000.

[8] J. Zhang and H. Xiao, "Computational studies on the infrared vibrational spectra, thermodynamic properties, detonation properties, and pyrolysis mechanism of octanitrocubane," Journal of Chemical Physics, vol. 116, no. 24, pp. 10674-10683, 2002.

[9] A. M. Astakhov, R. S. Stepanov, and A. Y. Babushkin, "On the detonation parameters of octanitrocubane," Combustion, Explosion and Shock Waves, vol. 34, no. 1, pp. 85-87, 1998.

[10] P. E. Eaton, M.-X. Zhang, R. Gilardi, N. Gelber, S. Iyer, and R. Surapaneni, "Octanitrocubane: a new nitrocarbon," Propellants, Explosives, Pyrotechnics, vol. 27, pp. 1-6, 2000.

[11] U. R. Nair, R. Sivabalan, G. M. Gore, M. Geetha, S. N. Asthana, and H. Singh, "Hexanitrohexaazaisowurtzitane (CL-20) and CL-20-based formulations," Combustion, Explosion and Shock Waves, vol. 41, no. 2, pp. 121-132, 2005.

[12] P. E. Eaton, R. L. Gilardi, and M.-X. Zhang, "Polynitrocubanes: advanced high-density, high-energy materials," Advanced Materials, vol. 12, no. 15, pp. 1143-1148, 2000.

[13] National Research Council of the National Academies, Advanced Energetic Materials, Committee on Advanced Energetic Materials and Manufacturing Technologies, Board on Manufacturing and Engineering Design, Division on Engineering and Physical Sciences, The National Academies Press, Washington, DC, USA, 2004.

[14] G. Zhou, J. Wang, W. D. He, N. B. Wong, A. Tian, and W. K. Li, "Theoretical investigation of four conformations of HNIW by B3LYP method," Journal of Molecular Structure, vol. 589-590, pp. 273-280, 2002.

[15] W. S. Ohlinger, P. E. Klunzinger, B. J. Deppmeier, and W. J. Hehre, "Efficient calculation of heats of formation," Journal of Physical Chemistry A, vol. 113, no. 10, pp. 2165-2175, 2009.

[16] S. W. Benson, F. R. Cruickshank, D. M. Golden et al., "Additivity rules for the estimation of thermochemical properties," Chemical Reviews, vol. 69, no. 3, pp. 279-324, 1969.

[17] S. W. Benson, Thermochemical Kinetics: Methods for the Estimation of Thermochemical Data and Rate Parameters, John Wiley \& Sons, New York, NY, USA, 2nd edition, 1976.

[18] G. Leroy, "The theoretical approach to some chemical problems," Advances in Quantum Chemistry, vol. 17, no. C, pp. 195, 1985.

[19] CHETAH- ASTM Computer Program for Chemical Thermodynamic and Energy Release Evaluation-CHETAH Version 8. 0, http://www.normas.com/ASTM/COMPs/CHETAH-9-0 .html.
[20] R. A. Pesce-Rodriguez, R. A. Fifer, K. L. McNesby et al., "Thermal decomposition of HNIW-based formulations," Technical Report BRL-TR-3402, AD-A255 613, 1991, http://oai.dtic.mil/oai/oai?verb=getRecord\&metadataPrefix= html\&identifier=ADA255613.

[21] M. F. Foltz, C. L. Coon, F. Garcia, and A. L. Nichols III, "Thermal stability of the polymorphs of hexanitrohexaazaisowurtzitane, Part I," Propellants, Explosives, Pyrotechnics, vol. 19, no. 1, pp. 19-25, 1994.

[22] M. F. Foltz, "Thermal stability of E-hexanitrohexaazaisowurtzitane in an Estane formulation," Propellants, Explosives, Pyrotechnics, vol. 19, no. 2, pp. 63-69, 1994.

[23] M. F. Foltz, C. L. Coon, F. Garcia, and A. L. Nichols III, "Thermal stability of the polymorphs of hexanitrohexaazaisowurtzitane, Part II," Propellants, Explosives, Pyrotechnics, vol. 19, no. 3, pp. 133-144, 1994.

[24] N. B. Bolotina, M. J. Hardie, R. L. Speer Jr., and A. A. Pinkerton, "Energetic materials: variable-temperature crystal structures of $\gamma$ - and $\varepsilon$-HNIW polymorphs," Journal of Applied Crystallography, vol. 37, no. 5, pp. 808-814, 2004.

[25] J. Li and T. B. Brill, "Kinetics of solid polymorphic phase transitions of CL-20," Propellants, Explosives, Pyrotechnics, vol. 32, no. 4, pp. 326-330, 2007.

[26] T. P. Russell, P. J. Miller, G. J. Piermarini, and S. Block, "Pressure/temperature phase diagram of hexanitrohexaazaisowurtzitane," Journal of Physical Chemistry, vol. 97, no. 9, pp. 1993-1997, 1993.

[27] S. Zeman and M. Krupka, "New aspects of impact reactivity of polynitro compounds, Part III. Impact sensitivity as a function of the imtermolecular interactions," Propellants, Explosives, Pyrotechnics, vol. 28, no. 6, pp. 301-307, 2003.

[28] X. W. Fan, X. H. Ju, Q. Y. Xia, and H. M. Xiao, "Strain energies of cubane derivatives with different substituent groups," Journal of Hazardous Materials, vol. 151, no. 1, pp. 255-260, 2008 


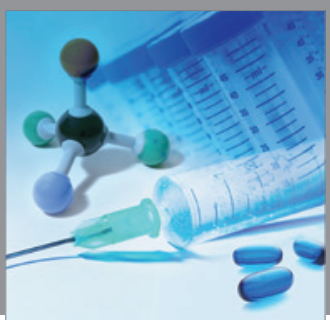

International Journal of

Medicinal Chemistry

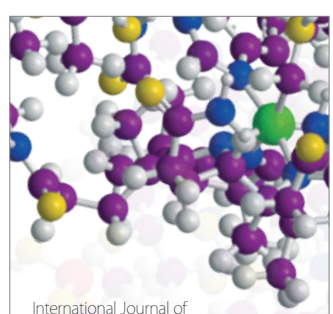

Carbohydrate Chemistry

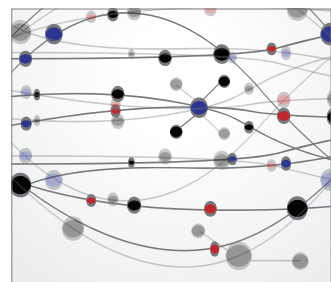

The Scientific World Journal
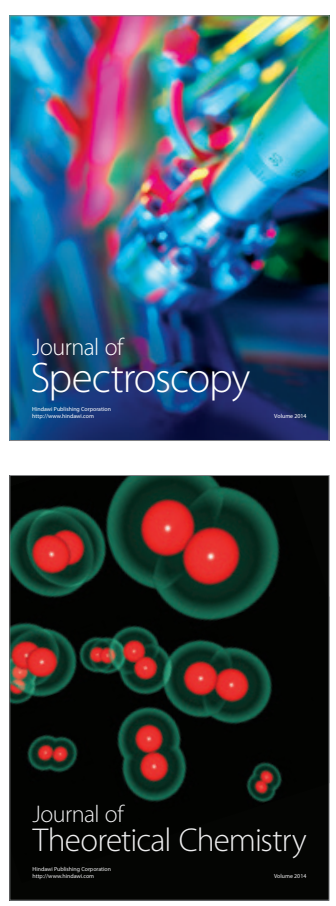
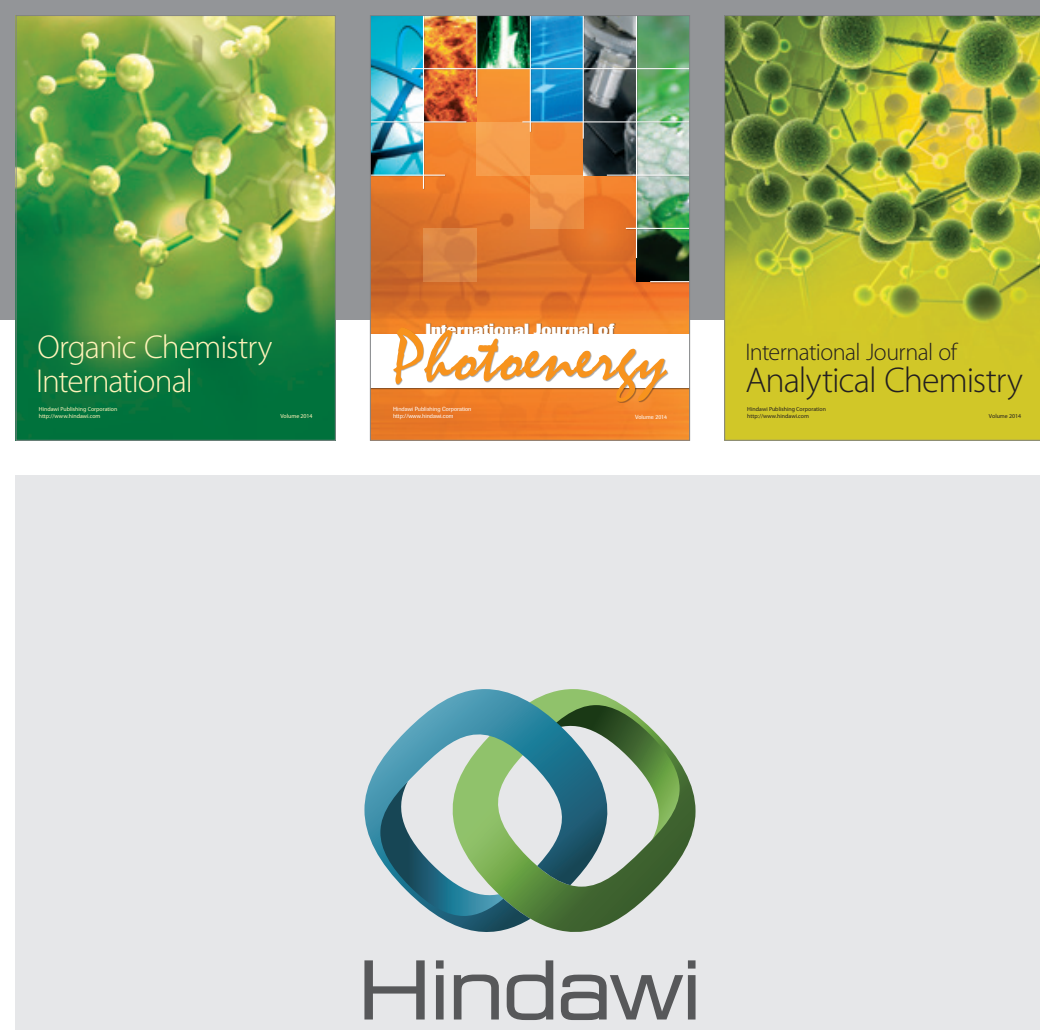

Submit your manuscripts at

http://www.hindawi.com
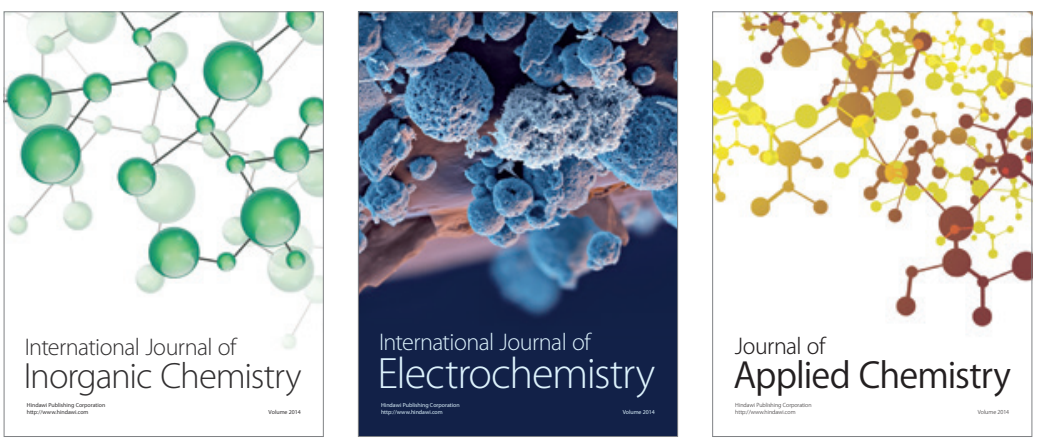

Journal of

Applied Chemistry
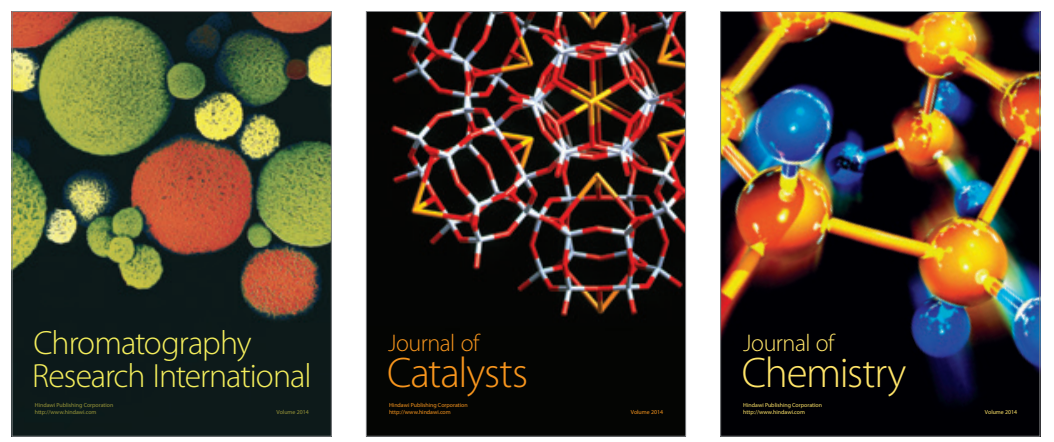
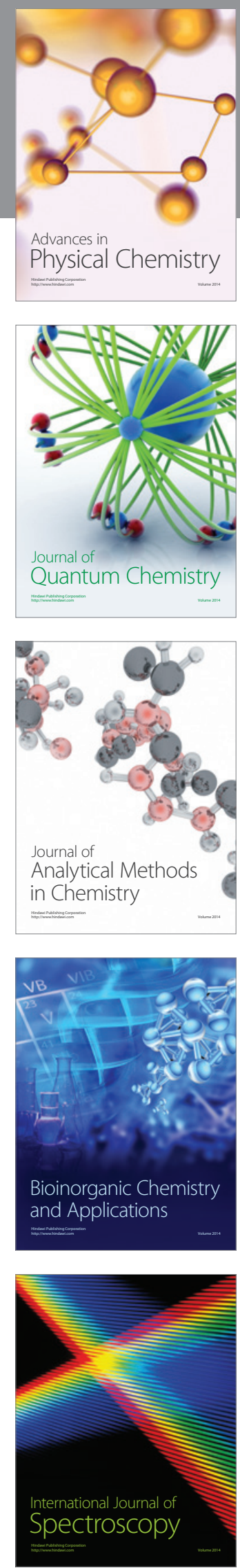